\title{
Utilizing Publicly Available Cancer Clinicogenomic Data on CBioPortal to Compare Epidermal Growth Factor Receptor Mutant and Wildtype Non-Small Cell Lung Cancer
}

\author{
Chirag Dhar ${ }^{1}$ \\ 1. Medicine and Cellular and Molecular Medicine, University of California San Diego, La Jolla, USA
}

Corresponding author: Chirag Dhar, chirag.dhar@gmail.com

\begin{abstract}
Publicly available clinicogenomic data on platforms such as the cancer BioPortal (cBioPortal.org) allow for efficient analyses by researchers with little or no experience working with Big Data. cBioPortal.org also allows for appropriate statistical testing and downloadable images for easy dissemination of findings. In this study, the cBioPortal.org platform was tested and its utility demonstrated by comparing cases of non-small cell lung cancer (NSCLC) with and without epidermal growth factor receptor gene (EGFR) mutations. Patients with EGFR mutations were more likely to be female, of Asian ethnicity, never-smokers, and be diagnosed with lung adenocarcinoma. Metastasis to the pleura, pleural fluid, and liver was common in patients with EGFR mutant NSCLC. On the other hand, lymph node, brain, and adrenal gland metastases were more common in patients with other mutations. While the median overall survival was about the same in the two groups, progression-free survival was significantly shorter in the EGFR mutant group. The mutational landscape was significantly different in the two groups with EGFR mutant NSCLCs having a lower mutational burden. Differences in copy number alterations between the two groups were also noted. The descriptive data generated from this study such as age, gender, smoking history, and histological subtype recapitulate findings of other studies on EGFR mutant NSCLCs. Further prospective and/or preclinical studies are needed to confirm differences noted in this study. cBioPortal.com queries may be used to supplement clinical/pre-clinical studies or to generate novel hypotheses.
\end{abstract}

Review began 04/14/2021 Review ended 04/22/2021 Published 04/25/2021

\section{(๑) Copyright 2021}

Dhar. This is an open access article distributed under the terms of the Creative Commons Attribution License CC-BY 4.0., which permits unrestricted use, distribution, and reproduction in any medium, provided the original author and source are credited.
Categories: Genetics, Oncology, Pulmonology

Keywords: anti-egfr, cbioportal, lung cancer, cancer genomics

\section{Introduction}

The establishment of cBioPortal.org, a central resource for patient- and sample-level clinicogenomic data in cancer, has allowed for in-depth analyses and comparisons of various cancer subtypes [1,2]. This platform provides fast and efficient analyses and is useful to researchers with little or no experience of working with large datasets. cBioPortal.org also has inbuilt functionality to perform relevant statistical tests and to download "publication quality" images. These outputs may then be used to support small clinical studies, pre-clinical findings, or even to generate novel hypotheses. To test and to demonstrate the utility of this platform, cBioPortal.org was queried for a common lung cancer mutation.

Lung cancers are one of the leading causes of cancer in both men and women and are a major cause of mortality in the American population with an anticipated 135,000 deaths in 2020 [3]. Eighty percent of all lung cancers are further categorized as non-small cell lung cancer (NSCLC) [4]. Major histological subtypes of NSCLC include lung adenocarcinoma, squamous cell carcinoma large cell carcinoma, adenosquamous cell carcinoma, and sarcomatoid carcinoma [5]. Recent advances in the clinicogenomics of lung cancer have uncovered the role of epidermal growth factor receptor (EGFR) mutations in a significant proportion of NSCLC patients [6]. EGFR is a member of the tyrosine kinase receptor family and plays an important role in cellular growth, proliferation, and signaling. Certain somatic EGFR mutations observed in a subset of NSCLC patients cause overamplification leading to constant activation and uncontrolled cell division [5]. Exons 18, 19, 20, and 21 of the EGFR gene are often the site of these mutations [7,8]. Some of the commonest EGFR mutations include inframe deletions of exon 19 and the exon 21 L858R point mutation [3]. These mutations are present in nearly a third of all lung adenocarcinomas and predict efficacy to EGFR tyrosine kinase inhibitors (TKIs) such as gefitinib and erlotinib [9]. Patients receiving TKIs have improved clinical outcomes as compared to those patients that receive conventional chemotherapy [10]. In this retrospective study, the clinicogenomic characteristics of EGFR mutant and EGFR wildtype NSCLC were compared and contrasted on the cBioPortal platform.

This article was previously posted on a preprint server:

https://www.medrxiv.org/content/10.1101/2020.08.07.20170027v3. 


\section{Materials And Methods}

The following schema (Figure 1) describes the methods of data collection and analysis in this retrospective cohort study. The references to studies utilized are provided here: [11-23].

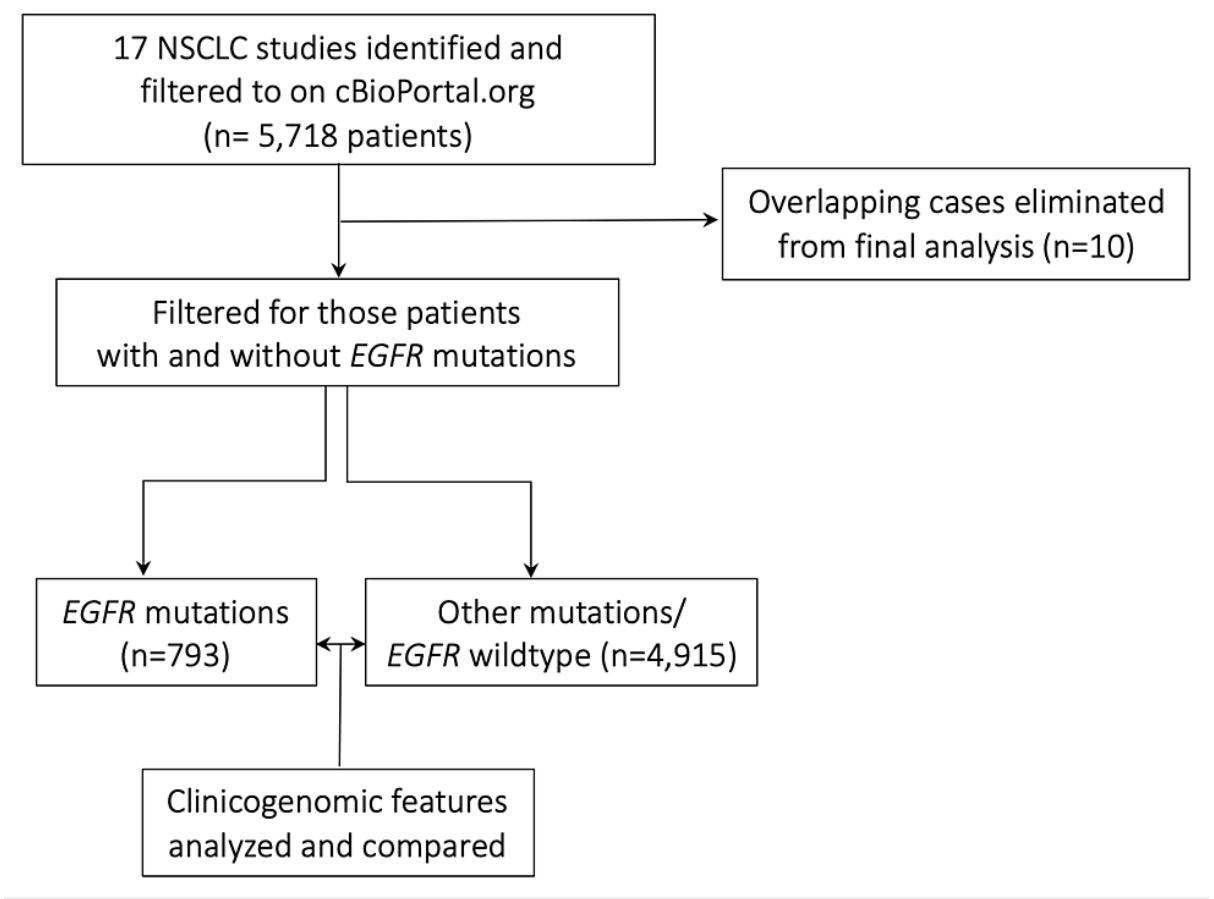

\section{FIGURE 1: Study schema}

A number of NSCLC studies $(n=17)$ were identified on cBioPortal.org that included a total of 5,718 patients. These cases were then divided in to those with and without EGFR mutations. cBioPortal classified 10 overlapping cases that were present in both groups likely because these patients had contributed multiple samples to these studies. After eliminating these overlapping cases, about $14 \%(n=793)$ were found to have EGFR mutations and the remaining $86 \%(\mathrm{n}=4,915)$ were found to have other mutations (EGFR wildtype). These two sub-groups were then compared by utilizing the cBioPortal.org environment and its functions.

NSCLC: non-small cell lung cancer; EGFR: epidermal growth factor receptor

Briefly, 17 cBioPortal.org NSCLC studies representing 5,718 patients were identified and filtered in September 2020. Of these, 803 were categorized to have EGFR mutations while the remaining 4,925 were not. A further analysis on the patient overlap function of cBioPortal.org revealed 10 cases that were present in both groups. These 10 patients possibly represent multiple samples that had different mutational patterns. For the purpose of this study, these overlapping samples were not analyzed. This elimination of overlapping samples was done to prevent the confounding effects of these cases being present in both groups. Also, inclusion of these samples would have biased some results towards the null.

Subsequently, clinicogenomic features of the two subgroups were compared utilizing the various functions available on cBioPortal.org. These analyses included a comparison of survival, staging, mutations, and copy number variations. Appropriate statistical tests/graphical representations as run by cBioPortal.org are included in the results. P- and O-values where appropriate are mentioned. The phrase "EGFR wildtype" and "other mutations" are used interchangeably to represent the sub-group that did not harbor EGFR mutations.

\section{Results}

\section{EGFR mutations were more common in women, patients of Asian ethnicity, never smokers, and those with lung adenocarcinoma}

Clinical characteristics of patients with EGFR mutations and those with other mutations (EGFR wildtype) were compared and are described in detail in Table 1 . The median age of diagnosis was slightly lower in patients with EGFR mutations. These patients were more likely to be never-smokers, women, of Asian ethnicity, and diagnosed with lung adenocarcinoma. These findings are in concert with those of other studies [24-28] on EGFR mutant NSCLC and suggest this cohort of more than five thousand patients is likely representative of differences seen in individual studies. 


\section{Cureus}

\begin{tabular}{|c|c|c|c|}
\hline Clinical characteristic & EGFR & Other mutations & P-value* \\
\hline Total number of subjects (\%) & $793(15.8)$ & 4195 (84.2) & - \\
\hline Median age at diagnosis in years & 64 & 67 & $<0.001$ \\
\hline Female sex (\%) & 64.5 & 41.4 & $<0.001$ \\
\hline Asian/Chinese ethnicity (\%) & 48.1 & 7.8 & $<0.001$ \\
\hline Never smokers (\%) & 38.9 & 7.9 & $<0.001$ \\
\hline Lung adenocarcinoma (\%) & 93.8 & 62.9 & $<0.001$ \\
\hline
\end{tabular}

\section{TABLE 1: Clinical characteristics of patients in the two subgroups}

The median age in the EGFR group was 64 years (range: 36-92, 25th percentile- 57, 75th percentile- 71 ) while in the other mutations group it was 67 years (range: $38-93,25$ th percentile- 59, 75th percentile- 73) with an absolute p-value by Kruskal Wallis test being $6.25 \times 10^{-10}$ and q-value of $6.07 \times$ $10^{-9}$. A Chi-squared statistic of 155.708 was obtained for the comparison of sex distribution in the two groups $\left(p-v a l u e\right.$ was $\left.<10^{-6}\right)$. Ethnicity data was available for 280 and 2,047 patients in the two groups with a significantly higher percentage being of Asian/Chinese ethnicity in the EGFR group (Chi-squared test statistic was 397.8601 , p-value $<10^{-6}$ ). Smoking histories of 403 and 2,221 patients was available in the two subgroups with never-smokers being significantly higher in the EGFR group (Chi-squared test statistic was $296.4889, \mathrm{p}$-value $<10^{-6}$ ). 744/805 EGFR cases and 3096/4915 other cases were lung adenocarcinoma (Chi-squared test statistic was 2755511, p-value $<10^{-6}$ ).

*all p-values reported to the third decimal place in the table.

\section{EGFR mutant cancers were more likely to metastasize than cancers} with other mutations

Figure 2 shows the differences in TNM and American Joint Committee on Cancer Code staging between EGFR mutant NSCLC and tumors with other mutations. EGFR mutant cancers were more likely to involve lymph nodes and to metastasize. EGFR mutant tumors were about as likely to be diagnosed as stage II, III, and IV as compared to tumors with other mutations.
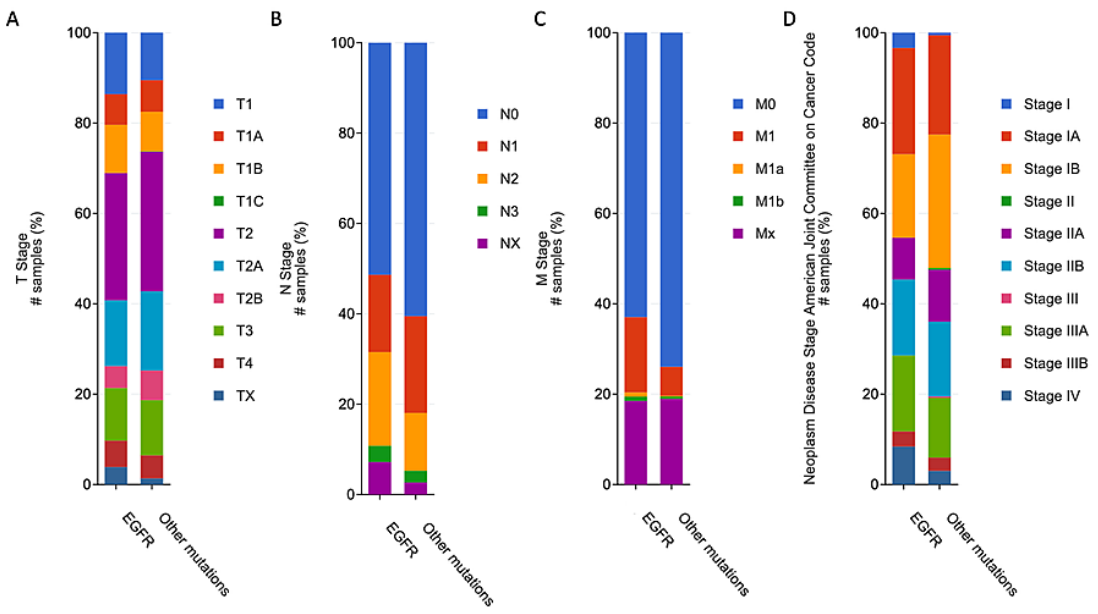

FIGURE 2: Graphical representation of differences in staging between EGFR mutant NSCLC and tumors with other mutations.

A) Differences in T stage: non-significant difference (Chi-squared test p-value 0.393); B) Differences in $\mathrm{N}$ stage: non-significant difference (Chi-squared test $p$-value 0.027 ); C) Differences in $M$ stage: non-significant difference (Chi-squared test p-value 0.254); D) Differences in American Joint Committee on Cancer Code staging: significant difference with Chi-squared test $p$-value of 0.00074 .

EGFR: epidermal growth factor receptor; NSCLC: non-small cell lung cancer 
Metastasis to pleura, pleural fluid, and liver was common in patients with EGFR mutant NSCLC, while metastasis to the lymph node, adrenal gland, and brain was more commonly associated with other mutations

Metastatic samples collected were used as a proxy to indicate frequency and sites of metastases. Metastatic samples were more frequently collected in patients with EGFR mutations as indicated by Figure $3 \mathrm{~A}$. Collected metastatic samples from pleura, pleural fluid, and liver were common in patients with EGFR mutant NSCLC, while metastatic samples from lymph nodes, adrenal gland, and brain were common in patients with other mutations (Figure $3 B$ ). These findings suggest a difference where EGFR mutant cancers are likely to metastasize.

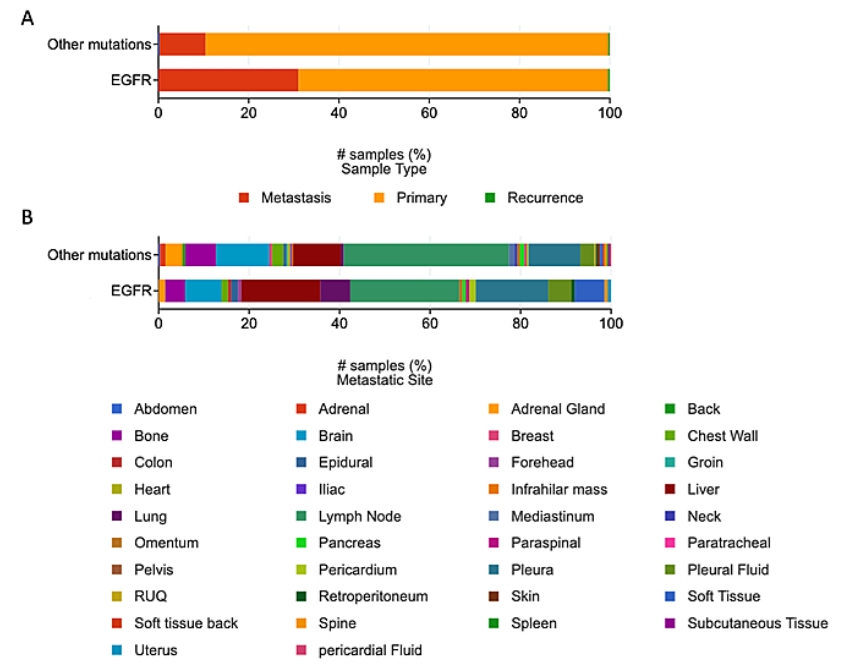

\section{FIGURE 3: Metastatic samples}

A) Frequency of metastatic sample collection: metastatic samples were likely to be collected from patients with EGFR mutations, though there might be a bias towards obtaining a biopsy in patients with metastatic EGFR NSCLC to direct targeted therapy; B) Frequency of metastatic sample collection by site: there was a significant difference between the two groups (Chi-squared test $p$-value 0.00641) with EGFR mutant samples more likely obtained from pleura, pleural fluid, and liver while metastatic deposits from the adrenal glands, lymph nodes, and brain were commonly collected from those with other mutations.

EGFR: epidermal growth factor receptor; NSCLC: non-small cell lunch cancer; RUQ: right upper quadrant

\section{Overall survival (OS) is comparable in the two groups while progression-free survival (PFS) is shorter in patients with EGFR mutations}

Overall survival and PFS were compared in the two groups and Kaplan-Meir curves were generated (Figure $4 A, 4 B)$. The OS in both the groups were comparable with EGFR mutant cancer patients having a median survival of $\sim 49$ months and those with other mutations surviving $\sim 53$ months (Figure $4 C$ ). The PFS in patients with EGFR mutations was significantly shorter than those with other mutations ( $\sim 16$ months versus $\sim 30$ months, Figure 4C). 


\section{Cureus}
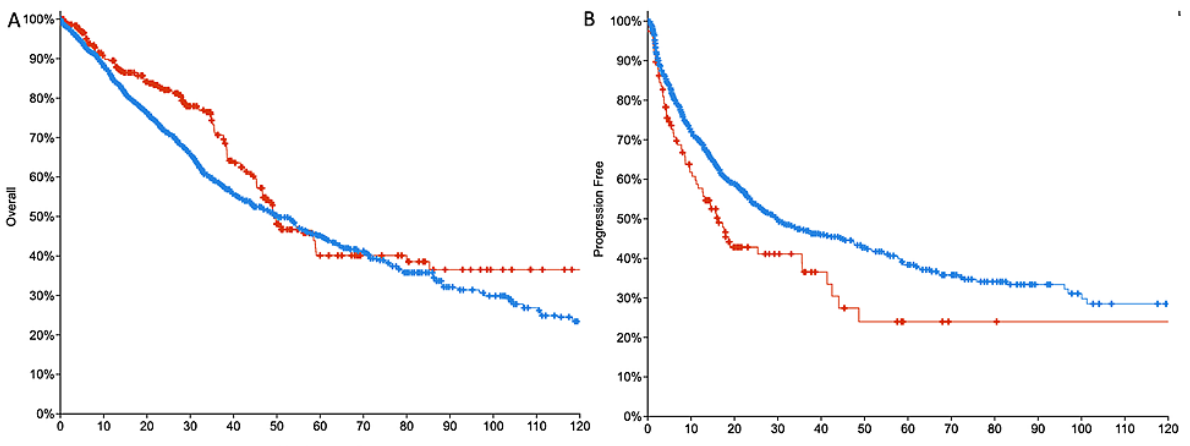

C

\begin{tabular}{|r|c|c|c|c|c|c|}
\hline & $\begin{array}{c}\text { Number } \\
\text { (OS) }\end{array}$ & $\begin{array}{c}\text { Deceased } \\
\text { (OS) }\end{array}$ & $\begin{array}{c}\text { Median } \\
\text { months (OS) }\end{array}$ & $\begin{array}{c}\text { Number } \\
\text { (PFS) }\end{array}$ & $\begin{array}{c}\text { Progressed } \\
\text { (PFS) }\end{array}$ & $\begin{array}{c}\text { Median } \\
\text { months (PFS) }\end{array}$ \\
\hline mEGFR & 389 & 127 & 49.25 & 120 & 66 & 16.34 \\
\hline mOther mutations & 3144 & 1146 & 50.24 & 1182 & 528 & 29.75 \\
\hline
\end{tabular}

\section{FIGURE 4: Survival analyses}

A) Overall survival (OS) Kaplan-Meir curve: logrank test p-value 0.0240; B) Progression-free survival (PFS) Kaplan-Meir curve: logrank test p-value 0.0042; C) Table depicting the number of patients where data was available, number that either died or progressed, and median survival in months.

EGFR: epidermal growth factor receptor

Patients with other mutations have a higher tumor mutation burden and have higher levels of cell-free DNA

The quantity of cell-free deoxyribonucleic acid (DNA) isolated from patients with mutations other than EGFR was higher than those with EGFR mutations (Figure $5 A$ ). Similarly, the tumor mutation burden was higher in patients with other mutations (Figure $5 B$ ).

A

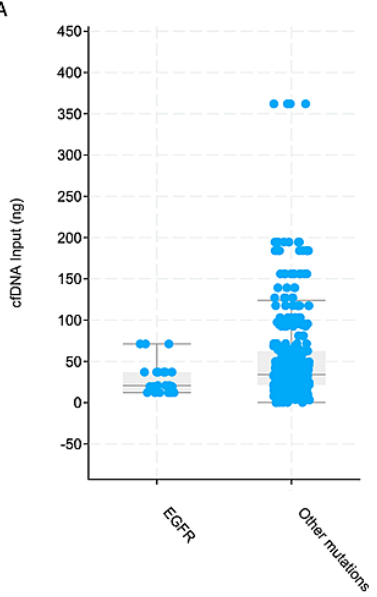

B

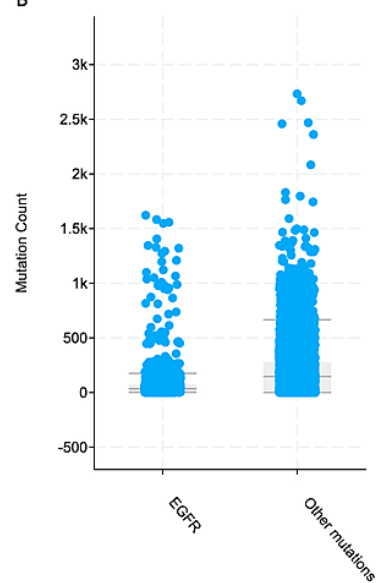

FIGURE 5: Cell-free DNA and tumor mutation count

A) Differences in quantity of cell-free DNA obtained from patients in the two groups: non-significant difference with median quantities being $20.7 \mathrm{ng}$ and $34 \mathrm{ng}$ in EGFR and other mutations group respectively (Chi-squared p-value 0.199); B) Differences in tumor mutation count: median mutation count of 35 in EGFR group versus 151 in other mutations group (significant difference with Kruskal Wallis test p-value $<10$ 10.

DNA: deoxyribonucleic acid; cfDNA: cell-free DNA; EGFR: epidermal growth factor receptor

Co-occurrence of mutations and copy number alterations differ in the 


\section{Cureus}

\section{two groups}

Commonly co-occurring mutations in the two groups were analyzed. TP53 mutations were amongst the commonest mutations in both groups and were excluded in this analysis. Similarly, as

anticipated EGFR mutations were most frequent in the EGFR mutant group but have been excluded in the analysis. The most frequently co-occurring mutations in the EGFR mutant group (with low frequency in the other group) were in SAP30L, DEFB4A, IL34, LRRC29, SPINK9, TTC1, REP15, CRIP2, CIAO2B, KRTCAP2, REXO5, SRP9, TNFRSF12A, CCL2, SH2D1B, AREG, HIST1H3F, TTC31, MRPL10, and SIAH1. The commonest mutations in patients with mutations other than EGFR were KRAS, RYR2, MUC16, CSMD3, USH2A, ZFHX4, KEAP1, SYNE1, STK11, NAV3, FLG, SPTA1, FAM135B, XIRP2, FAT3, RYR3, ZNF804A, KMT2C, CUBN, and SI. The frequency of occurrence of these mutations is depicted in Figure $6 A, 6 B$.

A

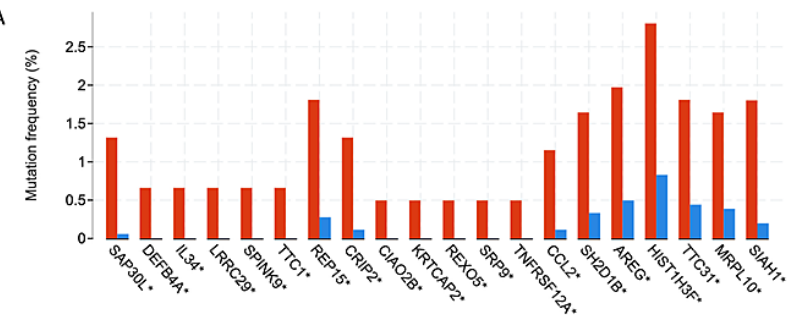

- EGFR

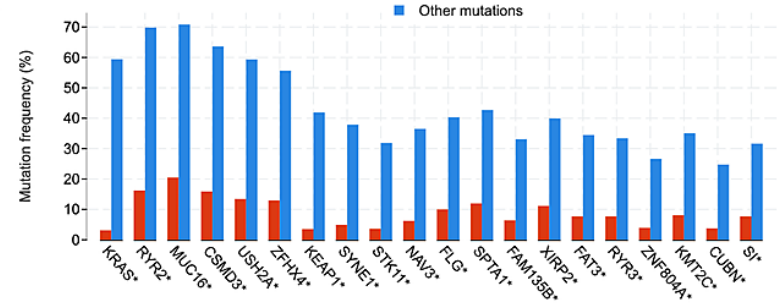

FIGURE 6: Frequency of commonest co-occurring mutations.

*asterisked genes represent significant differences

EGFR: epidermal growth factor receptor

The commonest copy number alterations were similarly analyzed. As was done with the mutations, EGFR amplifications have not been discussed as these are commonly seen in patients with EGFR mutations. The commonest amplifications in the EGFR group were on LOC650226, HPVC1, LOC100130849, DKFZP434L 192, CCT6A, SNORA15, SUMF2, VSTM2A-OT1, VOPP1, and PHKG1 (Figure 7A). The commonest alterations in the other group were amplifications of DCUN1D1, ATP11B, MCCC1, SOX2, B3GNT5, MCF2L2, LAMP3, KLHL24, and YEATS2 (Figure 7B). 
A

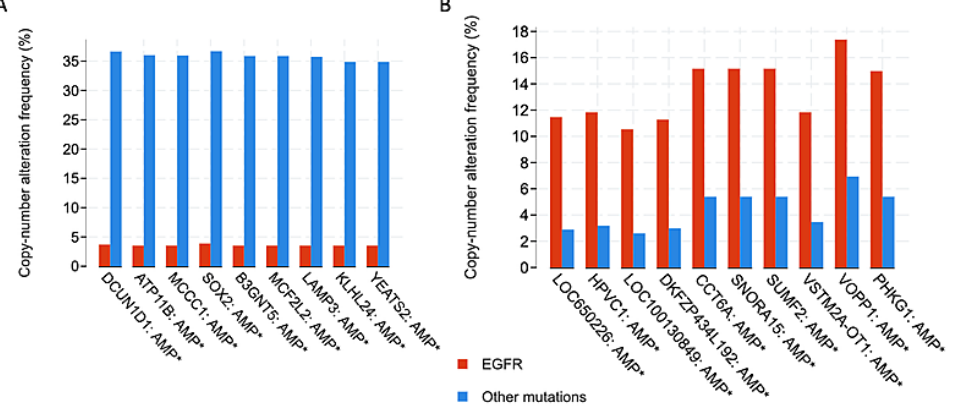

FIGURE 7: Frequency of various copy number alterations in the two groups

*asterisked genes represent significant differences

AMP: amplification; EGFR: epidermal growth factor receptor

\section{Discussion}

This retrospective study comparing EGFR mutant and EGFR wildtype NSCLC confirms the commonest clinical characteristics described in other studies. Patients with EGFR mutations were more likely to be female, never-smokers, and of Asian ethnicity. Lung adenocarcinoma was the commonest histological subtype in these patients. These findings being similar to other studies [24-27] pointed to these metadata being representative of differences seen in multiple individual studies. Further, the study showed that there were differences in the staging of cancer between the two groups with EGFR mutant cancer being more likely to be diagnosed at a more advanced stage. Metastatic samples collected from the two groups suggest that EGFR mutant cancers are more likely to metastasize to the pleura and pleural fluid as well as to the liver. On the other hand, NSCLC tumors harboring other mutations were more likely to metastasize to lymph nodes, the brain, and the adrenal glands. Likely due to these differences in staging and a higher likelihood of metastasis in the EGFR mutant group, the progression-free survival was shorter in patients with EGFR mutations. Yet, the difference in median overall survival was only 1 month. These findings are striking in light of targeted therapies for EGFR mutant cancers with EGFR TKIs and are strongly suggestive of rapid development of resistance to these therapies [29]. These can occur in the context of mutations such as T790M on exon [5].

At the genomic level, the tumor mutation burden was higher in EGFR wildtype patients. This finding is anticipated as these cancers may lack specific driver mutations and instead rely on multiple mutations for their transformation from normal tissue to cancer. The quantity of cell-free DNA, though not significantly different in the two groups, was lower in patients with EGFR mutations. The mutation and copy number alteration landscape were also different in the two groups and the commonest genes that were mutated or amplified are listed in the results section. These unique genomic signatures may be successfully exploited to predict EGFR TKI resistance early if confirmed in independent biomarker studies and/or cell-free DNA studies [30]. Prospective studies may show that some of these genomic features are present at the time of primary biopsy itself. Additionally, "bedside to bench" studies can be used to confirm these findings in cell lines and/or animal models which in turn could lead to studies on alternate "druggable” pathways.

Studies conducted on cBioPortal such as this one are limited by their retrospective design and are therefore likely to have a degree of convenience sampling. The effects of this error were likely minimal as the clinical characteristics of the two groups were similar to that seen in other prospective studies (age, sex, ethnicity, and smoking history were consistent with what has been reported in the literature). Additionally, there were limitations in the quality of data. For example, 6 of 17 included studies did not provide copy-number variation data. It is also possible that endpoints such as progression-free survival were calculated differently in the various studies included in these analyses. Despite filtering to NSCLC studies, a small number of lung cancers other than NSCLC $(<0.5 \%)$ also crept through the filters. This number was very small and likely did not alter the results significantly. cBioPortal data does not include specific treatment data and it is possible that a bias towards the null may have occurred in the overall survival data. Other studies have shown better outcomes in patients with EGFR mutations when treated with EGFR TKIs [7,10]. Another limitation that 
pertains to $\mathrm{cBioPortal}$ is the inability to run multivariate analysis to identify confounding factors and to adjust for these. cBioPortal does allow one to download the metadata and perform these analyses using other statistical software such as R or SPSS. For the convenience of this study, NSCLC was broadly divided into two groups: one for patients with EGFR mutations and the other for patients with wildtype EGFR. The second group is an oversimplification and includes a gamut of different mutations including some driver mutations such as KRAS. Finally, despite showing clear differences in the mutation and copy number alteration landscape in the two groups, this study does not clearly define differences in the molecular mechanisms.

\section{Conclusions}

Such studies on the clinicogenomic features of NSCLC and other cancers on cBioPortal are likely to throw light on possible new "druggable" targets. Additionally, hypotheses may be drawn from these studies and taken back to the "bench" to understand specific molecular mechanisms such as resistance to EGFR TKIs or the role of cell-free DNA. Future prospective studies and clinical trials are likely to include genomic level and transcriptomic analyses to draw broader clinicogenomic conclusions and lead to significant advances in the management of NSCLC.

\section{Additional Information \\ Disclosures}

Human subjects: Consent was obtained or waived by all participants in this study. Animal subjects: All authors have confirmed that this study did not involve animal subjects or tissue. Conflicts of interest: In compliance with the ICMJE uniform disclosure form, all authors declare the following: Payment/services info: All authors have declared that no financial support was received from any organization for the submitted work. Financial relationships: All authors have declared that they have no financial relationships at present or within the previous three years with any organizations that might have an interest in the submitted work. Other relationships: All authors have declared that there are no other relationships or activities that could appear to have influenced the submitted work.

\section{References}

1. Cerami E, Gao J, Dogrusoz U, et al.: The cBio cancer genomics portal: an open platform for exploring multidimensional cancer genomics data. Cancer Discov. 2012, 2:401-4. 10.1158/2159-8290.CD-12-0095

2. Gao J, Aksoy BA, Dogrusoz U, et al.: Integrative analysis of complex cancer genomics and clinical profiles using the cBioPortal. Sci Signal. 2013, 6:pl1. 10.1126/scisignal.2004088

3. Cancer Facts \& Figures. (2020). Accessed: August 7, 2020: https://www.cancer.org/research/cancer-factsstatistics/all-cancer-facts-figures/cancer-facts-figures-2020.html..

4. Zhang X, Zhao Y, Wang M, Yap WS, Chang AY: Detection and comparison of epidermal growth factor receptor mutations in cells and fluid of malignant pleural effusion in non-small cell lung cancer. Lung Cancer. 2008, 60:175-82. 10.1016/j.lungcan.2007.10.011

5. Cheng L, Alexander RE, Maclennan GT, et al.: Molecular pathology of lung cancer: key to personalized medicine. Mod Pathol. 2012, 25:347-69. 10.1038/modpathol.2011.215

6. Ding L, Getz G, Wheeler DA, et al.: Somatic mutations affect key pathways in lung adenocarcinoma . Nature. 2008, 455:1069-75. 10.1038/nature07423

7. Sharma SV, Bell DW, Settleman J, Haber DA: Epidermal growth factor receptor mutations in lung cancer Nat Rev Cancer. 2007, 7:169-81. 10.1038/nrc2088

8. D'Souza G, Dhar C, Kyalanoor V, Yadav L, Sharma M, Nawaz S M, Srivastava S: High frequency of exon 20 S768I EGFR mutation detected in malignant pleural effusions: a poor prognosticator of NSCLC. Cancer Rep (Hoboken). 2020, 3:e1262. 10.1002/cnr2.1262

9. Paez JG, Jänne PA, Lee JC, et al.: EGFR mutations in lung cancer: correlation with clinical response to gefitinib therapy. Science. 2004, 304:1497-500. 10.1126/science.1099314

10. Bell DW, Lynch TJ, Haserlat SM, et al.: Epidermal growth factor receptor mutations and gene amplification in non-small-cell lung cancer: molecular analysis of the IDEAL/INTACT gefitinib trials. J Clin Oncol. 2005, 23:8081-92. 10.1200/JCO.2005.02.7078

11. Thoracic PDX (MSK, provisional). Accessed: April 26, 2021: http://www.cbioportal.org/.

12. Yaeger R, Chatila WK, Lipsyc MD, et al.: Clinical sequencing defines the genomic landscape of metastatic colorectal cancer. Cancer Cell. 2018, 33:125-136.e3. 10.1016/j.ccell.2017.12.004

13. Jamal-Hanjani M, Wilson GA, McGranahan N, et al.: Tracking the evolution of non-small-cell lung cancer . N Engl J Med. 2017, 376:2109-21. 10.1056/NEJMoa1616288

14. Rizvi NA, Hellmann MD, Snyder A, et al.: Mutational landscape determines sensitivity to PD-1 blockade in non-small cell lung cancer. Science. 2015, 348:124-8. 10.1126/science.aaa1348

15. Gobbini E, Galetta D, Tiseo M, et al.: aMolecular profiling in Italian patients with advanced non-small-cell lung cancer: An observational prospective study. Lung Cancer. 2017, 111:30-7. 10.1016/j.lungcan.2017.06.009

16. Rizvi H, Sanchez-Vega F, La K, et al.: Molecular determinants of response to anti-programmed cell death (PD)-1 and anti-programmed death-ligand 1 (PD-L1) blockade in patients with non-small-cell lung cancer profiled with targeted next-generation sequencing. J Clin Oncol. 2018, 36:633-41. 10.1200/JCO.2017.75.3384

17. Campbell JD, Alexandrov A, Kim J, et al.: Distinct patterns of somatic genome alterations in lung adenocarcinomas and squamous cell carcinomas. Nat Genet. 2016, 48:607-16. 10.1038/ng.3564

18. Imielinski M, Berger AH, Hammerman PS, et al.: Mapping the hallmarks of lung adenocarcinoma with massively parallel sequencing. Cell. 2012, 150:1107-20. 10.1016/j.cell.2012.08.029 
19. Lung Adenocarcinoma, Lung Squamous Cell Carcinoma (TCGA, Firehose Legacy) . Accessed: April 26, 2021: http://cBioPortal.org.

20. The Cancer Genome Atlas Research Network, Disease Analysis Working Group, Collisson E et al.: Comprehensive molecular profiling of lung adenocarcinoma. Nature. 2014, 511:543-50. 10.1038/nature13385

21. TCGA PanCancer Atlas. Accessed: April 26, 2021: http://cBioPortal.org.

22. Jordan EJ, Kim HR, Arcila ME, et al.: Prospective comprehensive molecular characterization of lung adenocarcinomas for efficient patient matching to approved and emerging therapies. Cancer Discov. 2017, 7:596-609. 10.1158/2159-8290.CD-16-1337

23. Chen J, Yang H, Teo ASM, et al.: Genomic landscape of lung adenocarcinoma in East Asians . Nat Genet. 2020, 52:177-86. 10.1038/s41588-019-0569-6

24. Pao W, Miller V, Zakowski M, et al.: EGF receptor gene mutations are common in lung cancers from "never smokers" and are associated with sensitivity of tumors to gefitinib and erlotinib. Proc Natl Acad Sci U S A. 2004, 101:13306-11. 10.1073/pnas.0405220101

25. Lynch TJ, Bell DW, Sordella R, et al.: Activating mutations in the epidermal growth factor receptor underlying responsiveness of non-small-cell lung cancer to gefitinib. N Engl J Med. 2004, 350:2129-39. 10.1056/NEJMoa040938

26. Ciardiello F, Tortora G: EGFR antagonists in cancer treatment. N Engl J Med. 2008, 358:1160-74. 10.1056/NEJMra0707704

27. Riely GJ, Politi KA, Miller VA, Pao W: Update on epidermal growth factor receptor mutations in non-small cell lung cancer. Clin Cancer Res. 2006, 12:7232-41. 10.1158/1078-0432.CCR-06-0658

28. Zhou C, Wu Y-L, Chen G, et al.: Erlotinib versus chemotherapy as first-line treatment for patients with advanced EGFR mutation-positive non-small-cell lung cancer (OPTIMAL, CTONG- 0802): a multicentre, open-label, randomised, phase 3 study. Lancet Oncol. 2011, 12:735-42. 10.1016/S1470-2045(11)70184-X

29. Balak MN, Gong Y, Riely GJ, et al.: Novel D761Y and common secondary T790M mutations in epidermal growth factor receptor-mutant lung adenocarcinomas with acquired resistance to kinase inhibitors. Clin Cancer Res. 2006, 12:6494-501. 10.1158/1078-0432.CCR-06-1570

30. Suraj S, Dhar C, Srivastava S: Circulating nucleic acids: an analysis of their occurrence in malignancies . Biomed Rep. 2017, 6:8-14. 10.3892/br.2016.812 